\title{
Experimental study of Elementary Cellular Automata dynamics using the density parameter
}

\author{
Nazim Fatès 1 \\ ${ }^{1}$ ENS Lyon - LIP, \\ 46, allée d'Italie - 69364 Lyon Cedex 07 - France. \\ Nazim.Fateseens-lyon. fr
}

Classifying cellular automata in order to capture the notion of chaos algorithmically is a challenging problem than can be tackled in many ways. We here give a classification based on the computation of a macroscopic parameter, the $d$-spectrum, and show how our classifying scheme can be used to separate the chaotic ECA from the non-chaotic ones.

Keywords: Cellular Automata, Classification, Discrete Dynamical Systems, Density

\section{Introduction}

In [Gut89] Howard Gutowitz writes :

"A fundamental problem in theory of cellular automata is classification. A good classifi cation divides cellular automata into groups with meaningfully related properties. In general there are two types of classifi cations : phenotypic and genotypic. A phenotypic classifi cation divides a population into groups according to their observed behaviour. A genotypic classifi cation divides a population into groups according to the intrinsic structure of the entities in the population."

In 1984, the fi rst phenotypic classifi cation has been proposed by Wolfram [Wol84] dividing cellular automata (CA) according to their "observed dynamics". This classifi cation was very important since it motivated numerous researches but was at the same time criticised by many authors on grounds that no formal defi nitions of the classes were given.

In 1989, Culik and Yu in [ČPY89] proposed a formal defi nition of the classes according to some asymptotic behaviour of the cellular automaton and showed the undecidability of the classifying scheme.

The class of CA that has drawn much attention is the class of the 256, 2-state 3-neighbours CA, or so-called Elementary CA (ECA). An interesting axis of investigation was the application of topological analysis theory to cellular automata. In 1998, Cattaneo et al. in [CFM99] proposed a genotypic classifi cation of all of the $256 \mathrm{ECA}$ that has the advantage to rely on formal defi nitions as well as theoretical justifi cations. 
In this work, we propose a phenotypic classifi cation based on the observation of the statistical evolution of a "macroscopic parameter", the density, that can easily be computed and that is the result of the aggregation of the data obtained at the cell scale. We then compare the results obtained experimentally to Wolfram's and Cattaneo's classifi cation and examine the similarities and differences with both authors.

\section{Classifying ECA dynamics}

\subsection{Position of the problem}

The fi nite ECA have an advantage over CA with higher states or dimension : their dynamics can be easily represented using space-time diagrams in which the cell array $x(t)$ is represented by a horizontal sequence of light and dark cells that symbolise the states 0 and 1 and time as the vertical axis.

Fig. 1 represents 4 examples of space-time diagrams. Intuition leads to consider the fi rst three as very regular and the last one as more irregular, or more "chaotic".
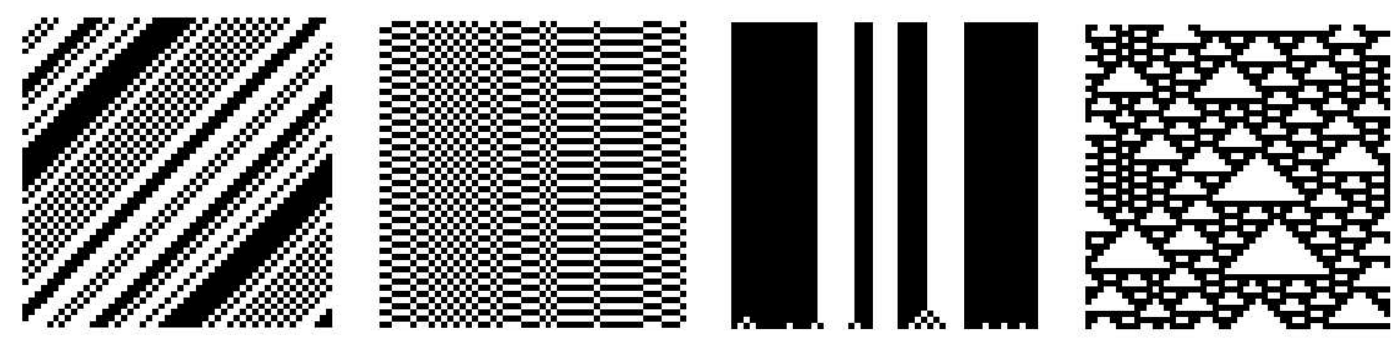

Fig. 1. Time goes from bottom to top, space-time diagrams are displayed for the rules $170,51,232$, 126 (see $\S 2.3$ for the rule encoding).

The purpose of our classifi cation is to discriminate the ECA that produce "chaotic looking" space-time diagrams from the others. From a theoretical point of vue, the work of Culik \& al. [ČPY89] showed that the problem of knowing, for a given CA, whether every (infi nite) confi guration evolves to a global (unique) fi xed point in a fi nite number of steps, is undecidable. A classifying scheme can thus be shown undecidable even with a classifi cation defi ned in a very simple an intuitive way.

From the work of Mazoyer and Rapaport [MR99], we know that it stills remains undecidable whether every circular (thus fi nite) confi guration of a given one-dimensional CA evolves to the same fi xed point. This result is ever more convincing as it shows that a classifi cation in which we demand a given propriety to be verifi ed for all of the confi gurations can be undecidable even when the classes are defi ned in very simple and intuitive ways.

An alternative approach, proposed in [Wol84] is to classify each ECA by looking at its behaviour and judging by eye whether most of the CA behaviour is periodic or chaotic. The goal of this work is to present a classifi cation which tries to algorithmically capture the informal notion of "most of the CA behaviour is periodic or chaotic" in order to have a classifying scheme that is effective and that can be implemented to test the 256 ECA. 


\subsection{The idea behind our classification}

To characterise formally the qualitative behaviour of a space-time diagram, several macroscopic parameters such as spatial or time entropy, Lyapunov exponent, etc. have been proposed. To our knowledge, none of them have been shown to be fully satisfying in regard to accounting for the "chaotic behaviour" of a CA. As far as the ECA space is concerned, we can say that we found very few tables in which the 256 ECA are exhaustively classifi ed according to the computation of a macroscopic parameter.

One diffi culty for establishing such an exhaustive study might be that the computation of a macroscopic parameter (e.g. entropy) is too slow and thus cannot be performed for a great number of initial conditions and for all the 256 ECA. The density parameter, i.e. the number of 1's divided by the total number of cells, is an effi ciently computed macroscopic parameter. We wondered whether it could be used to characterise the notion of chaos in the evolution of a CA.

An investigation of the statistical evolution of this parameter lead us to make the following observation:

- In a chaotic-looking ECA, for almost all of the initial conditions, the density parameter evolves with large statistical distributions.

- In a periodic-looking ECA, for almost all of the initial conditions, the evolution of the density stabilises after a transient time and enters into a cycle of small length, i.e. less or equal to 3.

This work is aimed at testing experimentally the validity of this observation and deriving a classifi cation of the ECA from it.

\subsection{General definitions}

The cellular automata we here study are objects which act on a one-dimensional fi nite lattice of cells, where each cell is assigned a state in $A=\{0,1\}$. Let $n$ be the size of the lattice, a configuration is an assignment of states to the cells of the lattice, it is a word in $A^{n}$, denoted $x=x_{0} \cdots x_{n-1}$, with $x_{i} \in A$. The set of all possible words of size $n, A^{n}$, is the phase space. The confi gurations are cyclic and all indices have to be considered in $\mathbb{Z} / n \mathbb{Z}$. Our fi nite $1 \mathrm{D}$ lattice is then a ring and we have $x_{1}=x_{n}$ and $x_{n}=x_{0}$.

An ECA is a function $f: A^{3} \rightarrow A$, called the local transition rule, to which we associate the global transition function $F_{f}: A^{n} \rightarrow A^{n}$ defi ned by :

$$
F_{f}\left(x_{0} \cdots x_{n-1}\right)=y_{0} \cdots y_{n-1}
$$

where

$$
y_{i}=f\left(x_{i-1}, x_{i}, x_{i+1}\right)
$$

Following [Wol84] we associate to each ECA $f$ its code :

$$
W(f)=f(0,0,0) \cdot 2^{0}+f(0,0,1) \cdot 2^{1}+\cdots+f(1,1,0) \cdot 2^{6}+f(1,1,1) \cdot 2^{7}
$$

An ECA $f$ having the code $R=W(f)$ is denoted by $R$ and we do not distinguish the rule from its code.

$\mathcal{D}=<F_{R}, A^{n}>$ is the discrete time dynamical system (DDS) associated to the rule $\mathrm{R}$ evolved on a ring of size $n$. We denote by $x(t)=F_{R}{ }^{t}(x)$ the $t^{t h}$ iterate of $F$ on $x$, with $x(0)=x$ and call an orbit the function $x(t)$.

Since the phase space is fi nite, every orbit is ultimately periodic, so:

$$
\exists T \in \mathbb{N}-\{0\}, \exists t_{0} \in \mathbb{N}, \forall t>t_{0}, x(t+T)=x(t)
$$




\subsection{Definition of the $d$-spectrum}

The density is the function $d: A^{n} \rightarrow[0,1]$ such that $d(x)=\frac{\#_{1}(x)}{n}$ where $\#_{1}(x)$ denotes the number of 1 in $x$ and $n$ is the size of the confi guration $x$.

For a given DDS $\mathcal{D}$ and a given orbit $x(t)$, we denote by $H=\{x(t)\}_{t \in \mathbb{N}}$ the set of reachable confi gurations in the orbit $x(t)$. The cycle of the orbit is the periodic part of $\mathrm{H}$, defi ned as the largest subset $C$ in $H$ whose image under the rule $F$ is invariant, i.e., such that $F(C)=C$. The transient time of the orbit, $T(x)$, is the size of the complement $T$ of $C$ in $H$.

The shift-invariant macroscopic parameter that we use as the discriminating factor of our classifi cation is called the $d$-spectrum of $x$, denoted by $\tau(x)$ and defi ned by:

$$
\tau(x)=|\{d(x), x \in C\}|
$$

It measures the number of different densities that appear in the cycle of an orbit. This number is clearly bounded by $n+1$, as :

$$
\forall x \in A^{n}, d(x) \in F_{n}=\left\{\frac{k}{n}, k \in[0, n]\right\}
$$

\section{Experimental protocol}

We present here the protocol we have used to perform the numerical experiments.

\section{1 $d$-spectrum estimation}

For a given DDS $\mathcal{D}=<F_{R}, A^{n}>$, we denote by $\tilde{\tau}(x)$ the numerical estimation of $\tau(x)$.

The estimation is performed by letting the dynamical system $\mathcal{D}$ evolve during $T_{\text {transient }}$ time steps and then measure the evolution of the density parameter during $T_{\text {sampling }}$ time steps. $T_{\text {transient }}$ and $T_{\text {sampling }}$ are two constants that are chosen as an approximation of the transient time $T(x)$ and the cycle length $|C|$. This results in the application of the following algorithm:

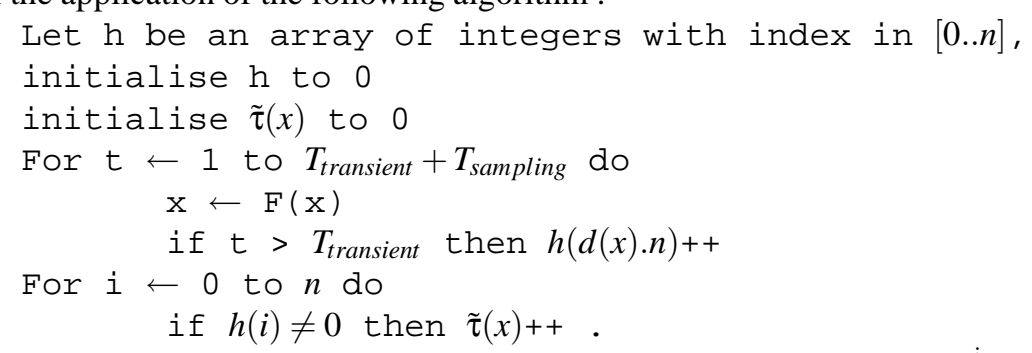

At the end, $h$ is the array containing in place $i$ the number of times density $\frac{i}{n}$ has been observed. The numerical $d$-spectrum $\tilde{\tau}(x)$ is computed as being the number of non-zero values of the histogram $h$.

\subsection{Class estimation}

In this work, we propose to estimate experimentally the number of different densities obtained in the orbits when starting from almost all of the initial conditions. Since the computation of all initial conditions is impossible in practice, we have to start from a limited set $I \subset A^{n}$ of initial conditions to perform the computation.

For a DDS $\mathcal{D}=<F_{R}, A^{n}>$, we construct the set $T_{I}=\left\{\tilde{\tau}\left(c_{i}\right), c_{i} \in I\right\}$ composed by all the $d$-spectra of the members of $I$. If our conjecture (see $\S 2.2$ ) is correct, then there should exist a constant $K_{p}$ such that 
all the values in $T_{I}$ are smaller than $K_{p}$ for a periodic-looking rule. Similarly, there should exist a constant $K_{c}$ such that $K_{c}>k_{p}$ and such that all the values in $T_{I}$ are greater than $K_{p}$ for a chaotic-looking rule.

We thus apply the following classifying scheme :

- $R$ is in class $\mathrm{P}$ if $T_{I} \subset\left[1, K_{p}\right]$,

- $R$ is in class $\mathrm{C}$ if $T_{I} \subset\left[K_{c}, \infty[\right.$,

- $R$ is in class $\mathrm{H}$ if it is neither in $\mathrm{P}$ nor in $\mathrm{C}$.

To test our conjecture, we expect the classifying algorithm to fulfi 1 the following requirements :

- The results can be obtained in a reasonable computing time.

- The algorithm produces the same results for different runs.

- The cardinal of the class $\mathrm{H}$ is small.

- The results are stable for different $n$ provided that $n$ is big enough.

So, we have to fi nd a set of initial conditions $I$, and two constants $K_{p}$ and $K_{c}$, such that the requirements stated above are verifi ed.

\subsection{How to fix the the constants}

\subsubsection{The case of $n, T_{\text {transient }}, T_{\text {sampling }}$}

The ring size $n$ should be chosen as large as possible in order to guarantee that the asymptotic behaviour of the orbits no longer depends on $n$. The limiting factor in the choice of $n$ is the computing abilities available for the numerical experiments. It has been shown experimentally that the values of the average transient time $T_{\text {transient }}$ and the average cycle length $|C|$ grow exponentially with $D$ for some chaotic-looking rules [Gut91].

We tune the value of $T_{\text {transient }}$ by ensuring that for all the periodic-looking ECA, for all initial conditions, the computed $\tilde{\tau}()$ is no longer varying for higher values of $T_{\text {transient }}$. While doing the experiments, we observed that the value of $T_{\text {sampling }}$ has little influence on the results as long as $T_{\text {sampling }} \geq n$, so we simply take $T_{\text {sampling }}=n$.

\subsubsection{The case of I}

Let us recall that the defi nition of our classifi cation is based on the estimation of the behaviour of a given rule on almost all of the initial conditions. We thus have to a assign a precise meaning to the expression "almost all". Clearly, we cannot take all the phase space $A^{n}$ as the set of initial conditions $I$ since we know that $A^{n}$ contains some confi gurations which behaviour is not dependent on the rule. However, we cannot exclude too many confi gurations in $A^{n}$ without taking the risk that $I$ is not representative of almost all the confi gurations of $A^{n}$. The criteria we take in this paper is to say that a set confi gurations $V_{n}$ defi ned for every $n$, is representative if $\lim _{n \rightarrow \infty} \frac{\left|V_{n}\right|}{2^{n}}=1$; i.e., if the limit set of $V_{n}$ has a measure 1 with the uniform measure.

For example, we can notice that whatever the rule and whatever the ring size, we have : $\tau(\overline{0}) \leq 2$, where $\overline{0}$ denotes the confi guration in which all cells are in state 0 . Thus, for a set of initial conditions $I$ 
containing the confi guration $\overline{0}$, any rule verifi es $1 \in T_{I}$ or $2 \in T_{I}$ (see $\S 3.2$ for the defi nition of $T_{I}$ ). This implies, according to our defi nitions of the classes, that no rule would ever be classifi ed in $\mathrm{C}$ if its sampling set $I$ contains $\overline{0}$. So, we have to exclude the confi gurations $\overline{0}$ and $\overline{1}$ from $I$ just as well as other special confi gurations.

In this work, we take the following set as a representative set of initial conditions:

$$
V_{n}=\left\{x \in A^{n}, 11 \subset x \text { and } 00 \subset x\right\}
$$

$V_{n}$ is the set of confi gurations in which not all the 1's or 0's are isolated. The motivation for using $\left(V_{n}\right)_{n \in \mathbb{N}}$ comes from the fact that for some ECA, such as rule 180, any confi guration in which all the 1s are isolated (surrounded by 0's) or all the 0's are isolated (surrounded by 1's) have a large $d$-spectrum indicating a chaotic behaviour, whereas all other confi gurations have small $d$-spectrum indicating a periodic behaviour. A more detailed description of such "strange" behaviours can be found in [CM98].

The most natural way of constructing $I$ is to select confi gurations randomly with a uniform sampling in $A^{n}$ and discard the confi gurations which are not in $V_{n}$. This would be correct and satisfying according to our criteria but we can notice that the greatest part of the selected confi gurations would have density close to $1 / 2$. In order to have different densities represented, we take a set of densities $D=\left\{d_{1}, d_{2}, \cdots, d_{k}\right\}$ and build $I$ as follows:

$$
I=\bigcup_{d \in D} I_{N_{s}}(d)
$$

where $I_{N_{s}}(d)$ denotes a subset of $V_{n}$, constructed with $N_{s}$ initial confi gurations of density $d$, that is words resulting from the concatenation of letters that have probability $d$ to be 1 and probability $1-d$ to be 0 .

\subsubsection{The case of $K_{p}, K_{c}$}

In order to guarantee that $\mathrm{P}$ and $\mathrm{C}$ are disjoint, we require that $K_{c}>K_{p}$. Recall that we have conjectured that $K_{p}=3$ would be suitable and that we want the class $\mathrm{H}$ to have a cardinal as small as possible.

\subsection{Results}

For $n=50$, it appears empirically that $T_{\text {transient }}=200$ was suitable in order to have the stability of the results. For the results here provided, we take $n=100$ and $T_{\text {transient }}=1000$.

Concerning the initial condition sampling, we always take 150 initial conditions for each ECA, with $D=\{0.3,0.4,0.5,0.6,0.7\}$ and $N_{s}=30$.

Because we conjectured that non-chaotic ECA would produce $d$-spectra less or equal to 3 , we take $K_{p}=3$ for all $n$. To detect the chaotic rules, we observe that taking $K_{c}=4$ for $n=50$ and $K_{c}=7$ for $n=100$ provides a classifi cation which fulfi ls the criteria of $\S 3.2$.

Using this numerical values, it appears that the output of the algorithm is stable when we run it several times. However, some particular rules (e.g rule 9) are sometimes classifi ed as $\mathrm{H}$ instead of P. This indicates that not all orbits enter into a cycle after 1000 steps and that $T_{\text {transient }}$ should be chosen bigger in order to guarantee the stability of the results. Unfortunately, we here reach the limit of our computing abilities as the number of elementary steps (application of the rule) is given by : $n_{o p}=256 \cdot N_{s} \cdot\left(T_{\text {transient }}+T_{\text {sampling }}\right) \cdot n=256 \cdot 150 \cdot 1200 \cdot 100 \sim 10^{10}$.

The numerical results are shown in the table at the end of the article. ECA are given with rule number $\mathrm{R}$, the smallest interval $T$ that contains $T_{I}$ and the class. We fi nd out that 204 ECA are P, 30 ECA are C 
and $22 \mathrm{ECA}$ are $\mathrm{H}$. This results confi rms that $K_{p}=3$ and $K_{p}=7$ is a suitable choice : $\mathrm{t}$ allows to fully discriminate $204+30$ ECA as $\mathrm{P}$ or C, letting the 22 ECA in $\mathrm{H}$ with an undetermined behaviour. Thus, a natural clusterisation of the ECA space seems to emerge.

\subsection{Symmetries of the ECA space}

One means of verifi cation of the consistence of the table is the use of symmetries. Indeed, for any ECA defi ned by local transition function $f$, we can associate the following ECA:

1. $f^{0}$, the reflected rule of $f$ defi ned by :

$$
\forall\left(x_{-1}, x_{0}, x_{1}\right) \in A^{3}, f^{0}\left(x_{-1}, x_{0}, x_{1}\right)=f\left(x_{1}, x_{0}, x_{-1}\right) .
$$

2. $f^{*}$, the conjugate rule of $f$ defi ned by :

$$
\forall\left(x_{-1}, x_{0}, x_{1}\right) \in A^{3}, f^{*}\left(x_{-1}, x_{0}, x_{1}\right)=\left[f\left(x_{-1}^{*}, x_{0}^{*}, x_{1}^{*}\right)\right]^{*} .
$$

where $*$ denotes the operation of changing 0 's into 1's and 1's into 0's.

3. $f^{0 *}$, the reflected conjugate rule of $f$ defi ned by :

$$
\forall\left(x_{-1}, x_{0}, x_{1}\right) \in A^{3}, f^{0 *}\left(x_{-1}, x_{0}, x_{1}\right)=\left[f\left(x_{1}^{*}, x_{0}^{*}, x_{-1}^{*}\right)\right]^{*} .
$$

As $f, f^{*}, f^{0}$ and $f^{0 *}$ are obtained using symmetries (they are isometrically conjugate), we expect that the classifying scheme attributes the same class to each of this rule. This requirement appears to be verifi ed experimentally with our classifying scheme.

\section{Analysis of the results}

We here compare the numerical results to two different classifi cations.

\subsection{Comparison with Cattaneo's et al. classification}

In [CFM99] Cattaneo et al. provide a table in which the 256 ECA are classifi ed according to the properties of their transition table. This genotypic classifi cation is based on topology and uses three main classes $C_{1}, C_{2}, C_{3}$ formally defi ned for an infinite one-dimensional lattice. The three main classes are subdivided into smaller classes using some particular proprieties of the local rule. Some of the defi ned sub-classes explicitly group rules that have the same asymptotic behaviour whereas some other sub-classes cannot allow qualitative predictions to be made.

For example, if we look at a sub-class which is characterised by "Periodic-1 attractor containing reachable $\overline{0} "$, we expect all the orbits to reach the $\overline{0}$ confi guration and to have a $d$-spectrum of 1 . Indeed, we fi nd that all rules of this subclass are P in our classifi cation (rules 72, 104, 200, 232, 4, 36, 132, 164).

Some other classes provided in Cattaneo's et al. classifi cation are not regrouping ECA that have a homogeneous behaviour. The most interesting case of non homogeneity appears for the rules 18, 22, 26, $82,94,122,126,146,182,218$ which are classifi ed in the same sub-class denoted "sub 90". Using our classifi cation, we fi nd that:

- rules $26,82,94$ are $\mathrm{H}$,

- rules $18,22,122,126,146,182$ are $\mathrm{C}$, 
- rule 218 is $\mathrm{P}$.

Indeed, one can verify experimentally that rule 218 seems to always converge (quickly) to a fi xed point. On the other hand rules 18,22, 122, 126, 146, 182 do exhibit a chaotic-looking behaviour. Rule 26 and 82 , which are isometrically conjugate, have a behaviour that cannot be clearly identifi ed as periodic or chaotic: the space-time diagrams it produces are reminiscent of the shift (rule 170 see fig. 1) but shows some kind of "complexity" (see fi g. 2). The rule 94 appears as an exception as it has a very stable signature having $T_{I} \subset[1,6]$ and could be classifi ed in $\mathrm{P}$ with $K_{p}=6$.

It thus appears that the predictions given by topological analysis are verifi ed experimentally and that the numerical results we provide might be useful to allow a fi ner analysis of the classes for which no theoretical prediction can be made.

Fig. 2. Some space-time diagrams obtained with the rules $26,41,54,73,94,110,154$ which represent the 7 symmetric-equivalent (see $\S 3.5$ ) hybrid rules.

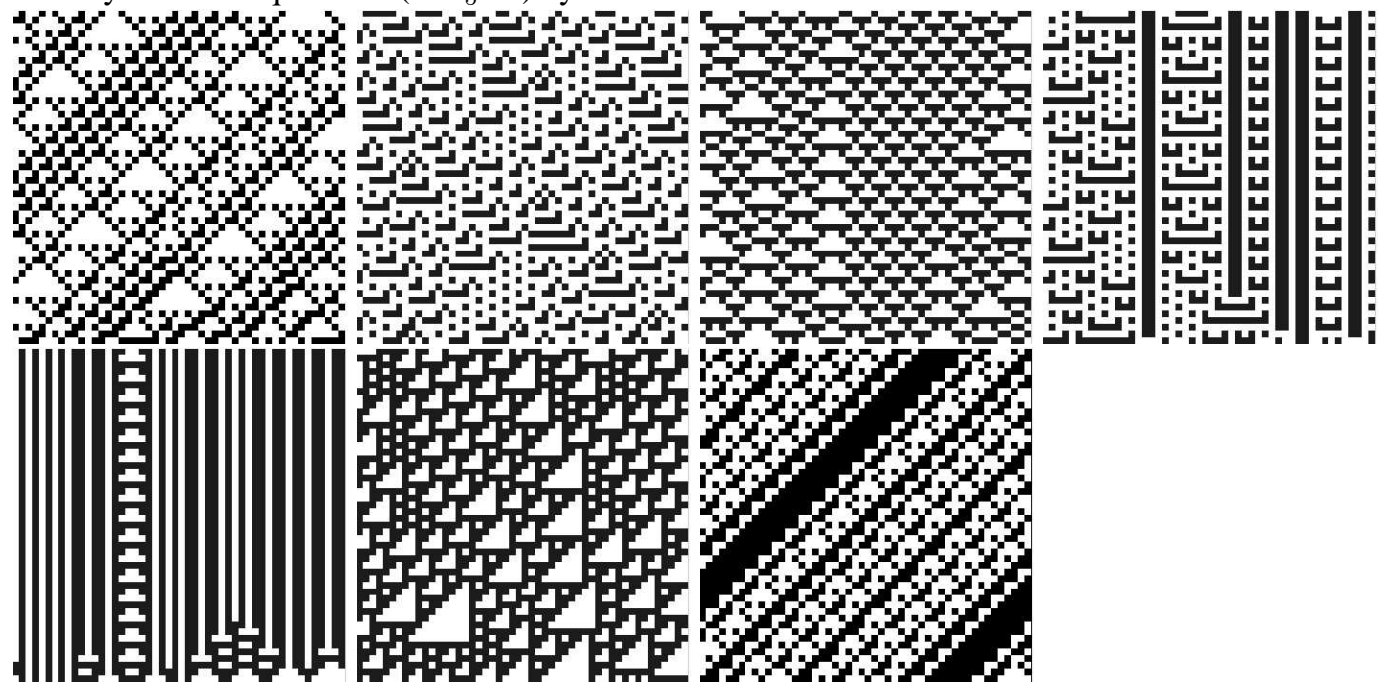

\subsection{Comparison with Wolfram's phenotypic classification}

The classifi cation proposed by Wolfram in [Wol84] divides the CA space into 4 classes :

\begin{tabular}{|l|r|}
\hline Class W1 & evolution of the CA leads to homogeneous stable confi guration \\
\hline Class W2 & evolution of the CA leads to a set of separated simple or periodic structures \\
\hline Class W3 & evolution of the CA leads to a chaotic pattern \\
\hline Class W4 & evolution leads to complex localised structures sometimes long-lived \\
\hline
\end{tabular}

Concerning class W4, which is the class of the most "complex" ECA, the fuzziness of the defi nition does not allow to classify an ECA as W4 without making a somehow arbitrary choice. It has been conjectured that class W4 is constituted out of the ECA that are capable of universal computation, that is, that are able to calculate the result of any function defi ned algorithmically. So far, only rule 110 (and its isometrically conjugated rules $137,124,193$ ) was clearly identifi ed having this ability (see [Wol02]). Rule 
54 is also another good candidate for having the computation universal ability but so far no evidence was given. As there is no "offi cial" Wolfram classifi cation, each author is allowed to make his own and we compared our results to the Wolfram classifi cation given in [CFM99].

We observe that that any ECA that we classify P is classifi ed W1 or W2, and that any ECA that we classify $\mathrm{C}$ is classifi ed W3. For the ECA that we classify H, we fi nd out that some of them, e.g rule 94, are $\mathrm{W} 2$ whereas some others, e.g rule 26 , are $\mathrm{W} 3$. This agreement confi rms the

It is worth noticing that rule 54 and rule 110 are both in class $\mathrm{H}$. Recall that our class $\mathrm{H}$ is defi ned as the class of ECA that have a set of $d$-spectrum that is neither all inside $\left[1, K_{p}\right]$ nor inside $\left[K_{c}, \infty[\right.$. Thus, an ECA which is $\mathrm{H}$ has the ability to produce small $d$-spectrum, like non-chaotic ECA, as well as large $d$ spectrum, like chaotic ECA. These particular ECA exhibit a behaviour that we can qualify to be "hybrid". Their characterisation using the $d$-spectrum might confi rm the idea that rules which exhibit a "complex" behaviour are situated in a transition zone in rule space, between chaotic ECA and ordered ECA [LPL90].

\section{Conclusion and perspectives}

We proposed to use an effi ciently computable macroscopic parameter, the $d$-spectrum, to classify the 256 rules of the ECA space. The most important features of the classifying scheme might be its coherence with other classifi cations and its ability to fi nd "complex" rules of the ECA space (rules 54 and 110).

Because we have worked on fi nite lattices, the classifi ciation operates on couples rule/lattice and might depend on the size of the lattice. In order to prove the membership of a rule for any ring size, it might be worth to formalise the classifi cation and to obtain theoretic results.

The characterisation of chaos we proposed for the ECA can be generalised to cellular automata with higher number of states and higher dimensions and even to objects which are not cellular automata such as boolean networks. One may wonder how the constants $T_{\text {sampling }}$ and $T_{\text {transient }}$ vary for such objects and whether a clusterisation of the space can still be exhibited. Another axis of investigation is to use this macroscopic parameter to characterise other concepts related to cellular automata, such as the "robustness" of a cellular automaton, that is its ability to keep its behaviour constant when submitted to little perturbation of its topology or synchronicity.

\section{References}

[CFM99] G. Cattaneo, E. Formenti, and L. Margara. Topological chaos and ca. In M. Delorme and J. Mazoyer, editors, Cellular Automata - A Parallel model, volume 460, pages 213-259. Kluwer Academic Publishers, 1999.

[CM98] G. Cattaneo and L. Margara. Generalized sub-shifts in elementary cellular automata: The "strange case" of chaotic rule 180. Theorectical Computer Science, 201(1-2):171-187, 1998.

[ČPY89] K. Čulik, Y. Pachl, and S. Yu. On the limit sets of cellular automata. SIAM Journal of Computing, 18:831-842, 1989.

[Gut89] H. Gutowitz. Mean fi eld vs. wolfram classifi cation of cellular automata (unpublished). Available on : http://www.santafe.edu/ xhag, 1989.

[Gut91] H. Gutowitz. Transients, cycles and complexity in cellular automata. Physical Review A, 44(12):7881-7884, December 1991. 
[LPL90] W. Li, N. Packard, and C. G. Langton. Transition phenomena in CA rule space. Physica D, 45:77, 1990.

[MR99] J. Mazoyer and I. Rapaport. Global fi xed points attractors of circular cellular automata and periodic tilings of the plane : undecidability results. Discrete Mathematics, 199:103-122, 1999.

[Wol84] S. Wolfram. Universality and complexity in cellular automata. Physica D, 10:1-35, 1984.

[Wol02] S. Wolfram. A new kind of science. Wolfram Media Inc., 2002.

Tab. 1: Experimental results for: $X=100, N_{s}=30, D=\{0.30, \cdots, 0.70\}, T_{\text {transient }}=1000, T_{\text {sampling }}=100, K_{p}=3, K_{c}=7$

\begin{tabular}{|c|l|l|l|c||c|l|l|l|l|l||c|l|l|l|l|l||c|c|c|c|c|}
\hline $\mathrm{R}$ & $\mathrm{T}$ & $\mathrm{P}$ & $\mathrm{H}$ & $\mathrm{C}$ & $\mathrm{R}$ & $\mathrm{T}$ & $\mathrm{P}$ & $\mathrm{H}$ & $\mathrm{C}$ & $\mathrm{R}$ & $\mathrm{T}$ & $\mathrm{P}$ & $\mathrm{H}$ & $\mathrm{C}$ & $\mathrm{R}$ & $\mathrm{T}$ & $\mathrm{P}$ & $\mathrm{H}$ & $\mathrm{C}$ \\
\hline 0 & {$[1,1]$} & $\mathrm{X}$ & & & 1 & {$[1,2]$} & $\mathrm{X}$ & & & 2 & {$[1,1]$} & $\mathrm{X}$ & & & 3 & {$[1,2]$} & $\mathrm{X}$ & & \\
4 & {$[1,1]$} & $\mathrm{X}$ & & & 5 & {$[2,2]$} & $\mathrm{X}$ & & & 6 & {$[1,2]$} & $\mathrm{X}$ & & & 7 & {$[1,2]$} & $\mathrm{X}$ & & \\
8 & {$[1,1]$} & $\mathrm{X}$ & & & 9 & {$[1,3]$} & $\mathrm{X}$ & & & 10 & {$[1,1]$} & $\mathrm{X}$ & & & 11 & {$[1,2]$} & $\mathrm{X}$ & & \\
12 & {$[1,1]$} & $\mathrm{X}$ & & & 13 & {$[1,1]$} & $\mathrm{X}$ & & & 14 & {$[1,2]$} & $\mathrm{X}$ & & & 15 & {$[1,2]$} & $\mathrm{X}$ & & \\
16 & {$[1,1]$} & $\mathrm{X}$ & & & 17 & {$[1,2]$} & $\mathrm{X}$ & & & 18 & {$[7,15]$} & & & $\mathrm{X}$ & 19 & {$[2,2]$} & $\mathrm{X}$ & & \\
20 & {$[1,2]$} & $\mathrm{X}$ & & & 21 & {$[1,2]$} & $\mathrm{X}$ & & & 22 & {$[12,35]$} & & & $\mathrm{X}$ & 23 & {$[1,2]$} & $\mathrm{X}$ & & \\
24 & {$[1,1]$} & $\mathrm{X}$ & & & 25 & {$[1,3]$} & $\mathrm{X}$ & & & 26 & {$[4,17]$} & & $\mathrm{X}$ & & 27 & {$[1,2]$} & $\mathrm{X}$ & & \\
28 & {$[1,2]$} & $\mathrm{X}$ & & & 29 & {$[1,2]$} & $\mathrm{X}$ & & & 30 & {$[18,28]$} & & & $\mathrm{X}$ & 31 & {$[2,2]$} & $\mathrm{X}$ & & \\
32 & {$[1,1]$} & $\mathrm{X}$ & & & 33 & {$[1,2]$} & $\mathrm{X}$ & & & 34 & {$[1,1]$} & $\mathrm{X}$ & & & 35 & {$[1,2]$} & $\mathrm{X}$ & & \\
36 & {$[1,1]$} & $\mathrm{X}$ & & & 37 & {$[1,2]$} & $\mathrm{X}$ & & & 38 & {$[1,2]$} & $\mathrm{X}$ & & & 39 & {$[1,2]$} & $\mathrm{X}$ & & \\
40 & {$[1,1]$} & $\mathrm{X}$ & & & 41 & {$[2,11]$} & & $\mathrm{X}$ & & 42 & {$[1,1]$} & $\mathrm{X}$ & & & 43 & {$[1,2]$} & $\mathrm{X}$ & & \\
44 & {$[1,1]$} & $\mathrm{X}$ & & & 45 & {$[18,29]$} & & & $\mathrm{X}$ & 46 & {$[1,1]$} & $\mathrm{X}$ & & & 47 & {$[1,2]$} & $\mathrm{X}$ & & \\
48 & {$[1,1]$} & $\mathrm{X}$ & & & 49 & {$[1,2]$} & $\mathrm{X}$ & & & 50 & {$[1,2]$} & $\mathrm{X}$ & & & 51 & {$[1,2]$} & $\mathrm{X}$ & & \\
52 & {$[1,2]$} & $\mathrm{X}$ & & & 53 & {$[1,2]$} & $\mathrm{X}$ & & & 54 & {$[2,51]$} & & $\mathrm{X}$ & & 55 & {$[1,2]$} & $\mathrm{X}$ & & \\
56 & {$[1,1]$} & $\mathrm{X}$ & & & 57 & {$[1,1]$} & $\mathrm{X}$ & & & 58 & {$[1,2]$} & $\mathrm{X}$ & & & 59 & {$[2,2]$} & $\mathrm{X}$ & & \\
60 & {$[9,16]$} & & & $\mathrm{X}$ & 61 & {$[1,3]$} & $\mathrm{X}$ & & & 62 & {$[1,3]$} & $\mathrm{X}$ & & & 63 & {$[1,2]$} & $\mathrm{X}$ & & \\
\hline
\end{tabular}




\begin{tabular}{|c|c|c|c|c|c|c|c|c|c|c|c|c|c|c|c|c|c|c|c|}
\hline $\mathrm{R}$ & $\mathrm{T}$ & $P$ & $\mathrm{H}$ & $\mathrm{C}$ & $\mathrm{R}$ & $\mathrm{T}$ & $P$ & $\mathrm{H}$ & $\mathrm{C}$ & $\mathrm{R}$ & $\mathrm{T}$ & $P$ & $\mathrm{H}$ & $\mathrm{C}$ & $\mathrm{R}$ & $\mathrm{T}$ & $P$ & $\mathrm{H}$ & $\mathrm{C}$ \\
\hline 64 & {$[1,1]$} & $\mathrm{X}$ & & & 65 & {$[1,3]$} & $\mathrm{X}$ & & & 66 & {$[1,1]$} & $\mathrm{X}$ & & & 67 & {$[1,3]$} & $\mathrm{X}$ & & \\
\hline 68 & {$[1,1]$} & $X$ & & & 69 & {$[1,1]$} & $\mathrm{X}$ & & & 70 & {$[1,2]$} & $\mathrm{X}$ & & & 71 & {$[1,2]$} & $\mathrm{X}$ & & \\
\hline 72 & {$[1,1]$} & X & & & 73 & {$[2,30]$} & & $\mathrm{X}$ & & 74 & {$[1,1]$} & $\mathrm{X}$ & & & 75 & {$[17,27]$} & & & $\mathrm{X}$ \\
\hline 76 & {$[1,1]$} & $X$ & & & 77 & {$[1,1]$} & $X$ & & & 78 & {$[1,1]$} & X & & & 79 & {$[1,1]$} & $\mathrm{X}$ & & \\
\hline 80 & {$[1,1]$} & $\mathrm{X}$ & & & 81 & {$[1,2]$} & $X$ & & & 82 & {$[3,17]$} & & X & & 83 & {$[1,2]$} & $\mathrm{X}$ & & \\
\hline 84 & {$[1,2]$} & $X$ & & & 85 & {$[1,2]$} & $X$ & & & 86 & {$[18,27]$} & & & X & 87 & {$[1,2]$} & $\mathrm{X}$ & & \\
\hline 88 & {$[1,1]$} & $X$ & & & 89 & {$[17,28]$} & & & $\mathrm{X}$ & 90 & {$[10,18]$} & & & X & 91 & {$[1,2]$} & $\mathrm{X}$ & & \\
\hline 92 & {$[1,1]$} & $\mathrm{X}$ & & & 93 & {$[1,1]$} & $\mathrm{X}$ & & & 94 & {$[1,6]$} & & $X$ & & 95 & {$[1,2]$} & $\mathrm{X}$ & & \\
\hline 96 & {$[1,1]$} & X & & & 97 & {$[2,4]$} & & X & & 98 & {$[1,1]$} & X & & & 99 & {$[1,1]$} & X & & \\
\hline 100 & {$[1,1]$} & $\mathrm{X}$ & & & 101 & {$[16,26]$} & & & $X$ & 102 & {$[11,17]$} & & & X & 103 & {$[1,3]$} & $\mathrm{X}$ & & \\
\hline 104 & {$[1,1]$} & $X$ & & & 105 & {$[10,17]$} & & & $X$ & 106 & {$[16,31]$} & & & X & 107 & {$[2,4]$} & & X & \\
\hline 108 & {$[1,2]$} & $X$ & & & 109 & {$[3,29]$} & & $\mathrm{X}$ & & 110 & {$[2,19]$} & & X & & 111 & {$[1,3]$} & $\mathrm{X}$ & & \\
\hline 112 & {$[1,1]$} & $X$ & & & 113 & {$[1,2]$} & $X$ & & & 114 & {$[1,2]$} & X & & & 115 & {$[1,2]$} & $\mathrm{X}$ & & \\
\hline 116 & {$[1,1]$} & $X$ & & & 117 & {$[1,2]$} & $X$ & & & 118 & {$[1,3]$} & X & & & 119 & {$[1,2]$} & $\mathrm{X}$ & & \\
\hline 120 & {$[17,29]$} & & & $\mathrm{X}$ & 121 & {$[3,4]$} & & X & & 122 & {$[7,28]$} & & & X & 123 & {$[1,2]$} & $\mathrm{X}$ & & \\
\hline 124 & {$[2,19]$} & & $\mathrm{X}$ & & 125 & {$[1,3]$} & $\mathrm{X}$ & & & 126 & {$[7,28]$} & & & $\mathrm{X}$ & 127 & {$[1,2]$} & $\mathrm{X}$ & & \\
\hline 128 & {$[1,1]$} & $\mathrm{X}$ & & & 129 & {$[7,30]$} & & & $\mathrm{X}$ & 130 & {$[1,1]$} & $\mathrm{X}$ & & & 131 & {$[1,3]$} & $\mathrm{X}$ & & \\
\hline 132 & {$[1,1]$} & $X$ & & & 133 & {$[1,6]$} & & X & & 134 & {$[1,2]$} & X & & & 135 & {$[17,28]$} & & & X \\
\hline 136 & {$[1,1]$} & $X$ & & & 137 & {$[2,18]$} & & $\mathrm{X}$ & & 138 & {$[1,1]$} & $X$ & & & 139 & {$[1,1]$} & $\mathrm{X}$ & & \\
\hline 140 & {$[1,1]$} & $\mathrm{X}$ & & & 141 & {$[1,1]$} & $X$ & & & 142 & {$[1,2]$} & X & & & 143 & {$[1,2]$} & $\mathrm{X}$ & & \\
\hline 144 & {$[1,1]$} & $X$ & & & 145 & {$[1,3]$} & $X$ & & & 146 & {$[7,14]$} & & & X & 147 & {$[2,51]$} & & X & \\
\hline 148 & {$[1,2]$} & $X$ & & & 149 & {$[18,30]$} & & & $X$ & 150 & {$[9,15]$} & & & X & 151 & {$[22,35]$} & & & X \\
\hline 152 & {$[1,1]$} & $\mathrm{X}$ & & & 153 & {$[10,18]$} & & & $X$ & 154 & {$[3,19]$} & & X & & 155 & {$[1,2]$} & $\mathrm{X}$ & & \\
\hline 156 & {$[1,2]$} & $X$ & & & 157 & {$[1,2]$} & $X$ & & & 158 & {$[1,2]$} & X & & & 159 & {$[1,2]$} & $\mathrm{X}$ & & \\
\hline 160 & {$[1,1]$} & $X$ & & & 161 & {$[7,28]$} & & & $\mathrm{X}$ & 162 & {$[1,1]$} & X & & & 163 & {$[1,2]$} & $\mathrm{X}$ & & \\
\hline 164 & {$[1,1]$} & $X$ & & & 165 & {$[9,18]$} & & & $X$ & 166 & {$[2,22]$} & & X & & 167 & {$[4,19]$} & & $X$ & \\
\hline 168 & {$[1,1]$} & $X$ & & & 169 & {$[16,29]$} & & & $X$ & 170 & {$[1,1]$} & X & & & 171 & {$[1,1]$} & $X$ & & \\
\hline 172 & {$[1,1]$} & $X$ & & & 173 & {$[1,1]$} & $X$ & & & 174 & {$[1,1]$} & X & & & 175 & {$[1,1]$} & $\mathrm{X}$ & & \\
\hline 176 & {$[1,1]$} & $X$ & & & 177 & {$[1,2]$} & $X$ & & & 178 & {$[1,2]$} & X & & & 179 & {$[1,2]$} & $\mathrm{X}$ & & \\
\hline 180 & {$[2,17]$} & & $X$ & & 181 & {$[3,14]$} & & X & & 182 & {$[7,15]$} & & & $X$ & 183 & {$[7,15]$} & & & $X$ \\
\hline 184 & {$[1,1]$} & $\mathrm{X}$ & & & 185 & {$[1,1]$} & $\mathrm{X}$ & & & 186 & {$[1,1]$} & $\mathrm{X}$ & & & 187 & {$[1,1]$} & $\mathrm{X}$ & & \\
\hline 188 & {$[1,1]$} & $\mathrm{X}$ & & & 189 & {$[1,1]$} & $\mathrm{X}$ & & & 190 & {$[1,1]$} & $X$ & & & 191 & {$[1,1]$} & $\mathrm{X}$ & & \\
\hline 192 & {$[1,1]$} & $X$ & & & 193 & {$[2,21]$} & & $\mathrm{X}$ & & 194 & {$[1,1]$} & X & & & 195 & {$[10,18]$} & & & $X$ \\
\hline 196 & {$[1,1]$} & $X$ & & & 197 & {$[1,1]$} & $X$ & & & 198 & {$[1,2]$} & X & & & 199 & {$[1,2]$} & $\mathrm{X}$ & & \\
\hline 200 & {$[1,1]$} & $X$ & & & 201 & {$[1,2]$} & $X$ & & & 202 & {$[1,1]$} & X & & & 203 & {$[1,1]$} & $\mathrm{X}$ & & \\
\hline 204 & {$[1,1]$} & $X$ & & & 205 & {$[1,1]$} & X & & & 206 & {$[1,1]$} & X & & & 207 & {$[1,1]$} & $\mathrm{X}$ & & \\
\hline 208 & {$[1,1]$} & $\mathrm{X}$ & & & 209 & {$[1,1]$} & $\mathrm{X}$ & & & 210 & {$[2,15]$} & & $X$ & & 211 & {$[1,2]$} & $\mathrm{X}$ & & \\
\hline 212 & {$[1,2]$} & $X$ & & & 213 & {$[1,2]$} & $X$ & & & 214 & {$[1,2]$} & X & & & 215 & {$[1,2]$} & $\mathrm{X}$ & & \\
\hline 216 & {$[1,1]$} & $X$ & & & 217 & {$[1,1]$} & $X$ & & & 218 & {$[1,1]$} & X & & & 219 & {$[1,1]$} & $\mathrm{X}$ & & \\
\hline 220 & {$[1,1]$} & X & & & 221 & {$[1,1]$} & $\mathrm{X}$ & & & 222 & {$[1,1]$} & X & & & 223 & {$[1,1]$} & $\mathrm{X}$ & & \\
\hline 224 & {$[1,1]$} & $X$ & & & 225 & {$[16,29]$} & & & $X$ & 226 & {$[1,1]$} & X & & & 227 & {$[1,1]$} & $\mathrm{X}$ & & \\
\hline 228 & {$[1,1]$} & $X$ & & & 229 & {$[1,1]$} & $\mathrm{X}$ & & & 230 & {$[1,1]$} & $X$ & & & 231 & {$[1,1]$} & $\mathrm{X}$ & & \\
\hline 232 & {$[1,1]$} & $X$ & & & 233 & {$[1,1]$} & $X$ & & & 234 & {$[1,1]$} & X & & & 235 & {$[1,1]$} & $\mathrm{X}$ & & \\
\hline 236 & {$[1,1]$} & $X$ & & & 237 & {$[1,1]$} & $\mathrm{X}$ & & & 238 & {$[1,1]$} & X & & & 239 & {$[1,1]$} & $\mathrm{X}$ & & \\
\hline 240 & {$[1,1]$} & $\mathrm{X}$ & & & 241 & {$[1,1]$} & $\mathrm{X}$ & & & 242 & {$[1,1]$} & X & & & 243 & {$[1,1]$} & $\mathrm{X}$ & & \\
\hline 244 & {$[1,1]$} & $X$ & & & 245 & {$[1,1]$} & $\mathrm{X}$ & & & 246 & {$[1,1]$} & X & & & 247 & {$[1,1]$} & $\mathrm{X}$ & & \\
\hline 248 & {$[1,1]$} & $\mathrm{X}$ & & & 249 & {$[1,1]$} & $\mathrm{X}$ & & & 250 & {$[1,1]$} & X & & & 251 & {$[1,1]$} & $\mathrm{X}$ & & \\
\hline 252 & {$[1,1]$} & $X$ & & & 253 & {$[1,1]$} & $X$ & & & 254 & {$[1,1]$} & X & & & 255 & {$[1,1]$} & X & & \\
\hline
\end{tabular}


